\title{
Hyperparameter Optimization of Two-Hidden-Layer Neural Networks for Power Amplifiers Behavioral Modeling Using Genetic Algorithms
}

\author{
Siqi Wang, Morgan Roger, Julien Sarrazin, Member, IEEE, and Caroline Lelandais-Perrault
}

\begin{abstract}
Neural networks (NN) are efficient techniques for behavioral modeling of power amplifiers (PA). This paper proposes a genetic algorithm to determine the optimal hyperparameters of the NN model for a PA. Different activation functions are compared. The necessary number of training epochs is also studied to get an optimal solution with a significantly reduced computational complexity. Experimental measurements on a PA with different signals validate the $\mathrm{NN}$ models determined by the proposed method.
\end{abstract}

Index Terms-Behavioral modeling, genetic algorithm, neural networks, nonlinear distortion, power amplifiers

\section{INTRODUCTION}

$\mathbf{T}$ HE POWER amplifiers (PA) performance is crucial in modern wireless communication systems [1]. Modeling the behavior of the PA is crucial to estimate and analyze its performance [2]-[4].

Neural networks $(\mathrm{NN})$ have been widely used in PA behavioral modeling with similar or better performance compared with classical analytic models [4]-[9]. A real-valued timedelay NN model has been proposed in [5] by decomposing the baseband input signal into in-phase and quadrature component (I/Q) signals. The output signal is buffered to the input layer in [6], which improved the modeling accuracy. Instead, authors in [7] added nonlinear terms of the input signal to the input layer. Recently, some studies [8], [9] applied NN models with two or more hidden layers for better modeling accuracy.

The memory depth, the number of layers and the number of hidden neurons in each layer have a great impact on the performance of the NN model [10]. They are often determined in an empirical way or by sweeping search [6], [7].

Some optimization algorithms, such as hill-climbing [11], [12] and particle swarm optimization [13], have been applied to determine the optimal structure of a Volterra-series-based model. However, the hyperparameter optimization of the NN has always been a challenge.

Manuscript received xxx, 2019; accepted xxx, 2019. (Corresponding author: S. Wang.)

S. Wang, M. Roger, C. Lelandais-Perrault are with GeePs - Group of electrical engineering - Paris, CNRS, CentraleSupélec, Univ. Paris-Sud, Université Paris-Saclay, Sorbonne Université, 3 \& 11 rue Joliot-Curie, Plateau de Moulon 91192 Gif-sur-Yvette CEDEX, France (e-mail of authors: firstname.surname@centralesupelec.fr).

J. Sarrazin is with the Sorbonne Université, Laboratory of Electronics and Electromagnetism, L2E, F-75005 Paris, France (e-mail: julien.sarrazin@upmc.fr).

Color versions of one or more of the figures in this paper are available online at http://ieeexplore.ieee.org.

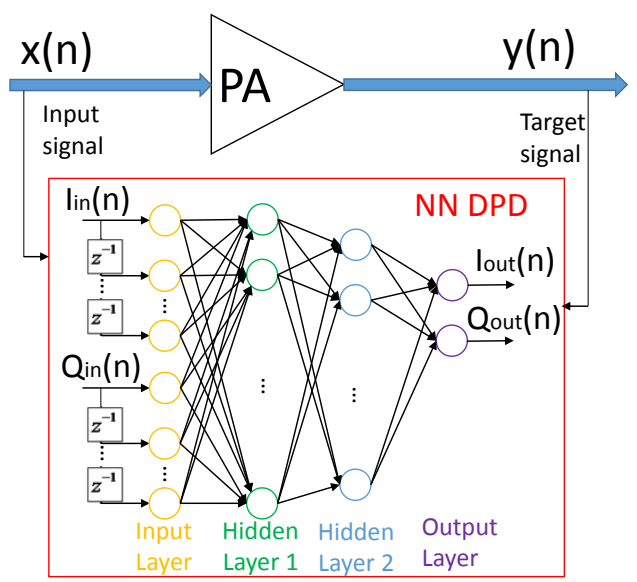

Fig. 1. PA behavioral modeling with neural network

In this paper, we propose to optimize the hyperparameters of a two-hidden-layer NN using genetic algorithms (GA). To the authors' knowledge, this is the first time that the $\mathrm{NN}$ hyperparameter problem for the PA behavioral modeling is addressed. The number of training epochs is optimized to reduce the running time.

\section{Multi-Layer Neural Network Model}

The PA modeling system architecture is illustrated in Fig 1. In order to train the network, we take the PA input signal $x(n)$ as the NN input signal and the PA output signal $y(n)$ as the target signal. They are decomposed into real-valued I/Q signals:

$$
\begin{aligned}
& x(n)=I_{\text {in }}(n)+i Q_{\text {in }}(n) \\
& y(n)=I_{\text {out }}(n)+i Q_{\text {out }}(n)
\end{aligned}
$$

The input layer is composed of $I_{\mathrm{in}}(n), Q_{\mathrm{in}}(n)$ and their delayed samples. The memory depth is $L$ which corresponds to the number of delay taps. We denote the number of neurons in the $l$-th hidden layers by $N_{l}$. The output $s_{k(l)}$ of the $k$-th neuron in the $l$-th layer can be then expressed as a function of the inputs $t_{j}$ :

$$
s_{k(l)}=f\left(\sum_{j=1}^{N_{l-1}} w_{j k} t_{j}+b_{k}\right)
$$

where $w_{j k}$ is the weight, $b_{k}$ is the bias, $f(\cdot)$ is the activation function. In this paper, we test two different activation functions: tangent sigmoid (tansig) and rectified linear unit (ReLU) 
as follows [9]:

$$
\begin{aligned}
& \operatorname{tansig}(x)=\frac{1-e^{-2 x}}{1+e^{-2 x}} \\
& \operatorname{ReLU}(x)= \begin{cases}x & \text { if } x \geq 0 \\
0 & \text { otherwise. }\end{cases}
\end{aligned}
$$

The activation function of the output layer is linear.

The training process is realized by backward computation using the Levenberg-Marquardt algorithm [14]. The input and target signals are randomly divided into 3 segments for training, validating and testing. The process can be composed of 2 phases:

1) At each epoch, we take the first segment to compute the values of $w_{j k}$ and $b_{k}$ and validate the computed NN with the second segment.

2) After the training, we test the $\mathrm{NN}$ with the third segment.

The modeling performance is evaluated with the normalized mean square error (NMSE) between the measured PA output $y(n)$ and the model output $\hat{y}(n)$ :

$$
\mathrm{NMSE}_{\mathrm{dB}}=10 \log _{10}\left[\frac{\sum_{n}|y(n)-\hat{y}(n)|^{2}}{\sum_{n}|y(n)|^{2}}\right] .
$$

The hyperparameters of the two-hidden-layer NN model to be optimized in this paper are: the memory depth $L$, the number of neurons in the first and the second hidden layer $N_{1}$ and $N_{2}$ respectively.

\section{GENETIC ALGORITHM}

Genetic algorithms are iterative stochastic search mechanisms based on the idea of Darwinian natural selection theory. After generational evolution, better genes are kept. In this algorithm, a fitness is defined to evaluate whether a gene is good. In our implementation, the genes are represented by NN hyperparameters $\left(L, N_{1}, N_{2}\right)$ in binary bits. We train the corresponding NN model to evaluate its fitness which is given by the error defined in (5) between the target signal and the output of NN model in the test phase (phase 2 in Section II). We use $\mathbf{P}$ to denote the group of parents and $q$ as the number of generations. The maximum generation number is set to $Q$.

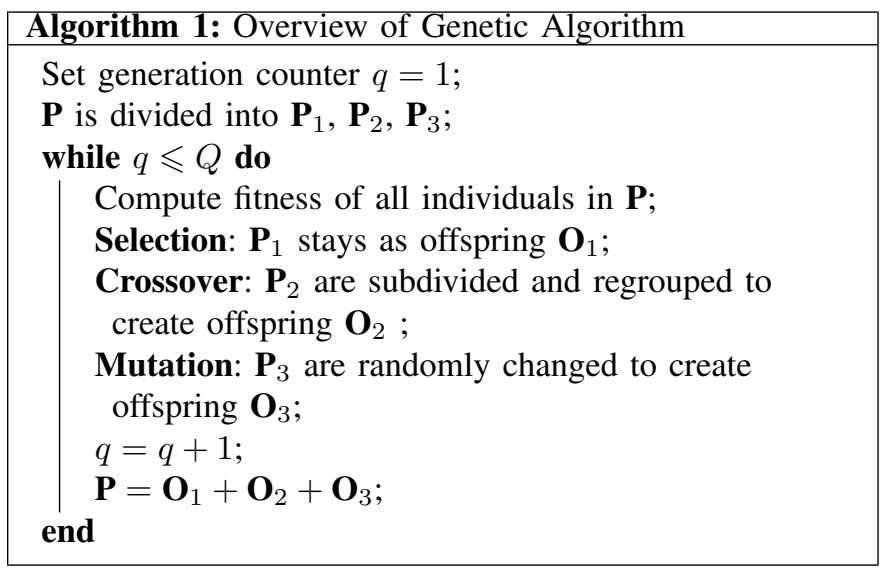

The offspring of each generation is created by the parents of the previous generation through selection, crossover and

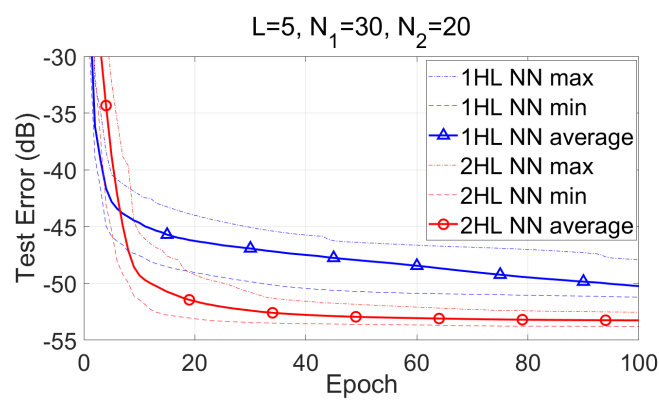

Fig. 2. Test error vs Epoch in training NN model with tansig as activation function, HL NN: hidden-layer neural network

mutation as described in Algorithm 1. The parents are listed from the best to the worst and then divided into three groups: the first group $\mathbf{P}_{1}$ has the best $5 \%$ individuals, the second group $\mathbf{P}_{2}$ has the best $80 \%$ of the remaining individuals, and the third group $\mathbf{P}_{3}$ has the rest. Selection: we keep $\mathbf{P}_{1}$ completely into the following generation as the first offspring group $\mathbf{O}_{1(q)}$. Crossover: we mix the genes of $\mathbf{P}_{2}$ to constitute the second offspring group $\mathbf{O}_{2(q)}$. Mutation: we randomly change the genes of $\mathbf{P}_{3}$ and create the third offspring group $\mathbf{O}_{3(q)}$. The parents of the following generation $\mathbf{P}_{(q+1)}$ is the union of $\mathbf{O}_{1(q)}, \mathbf{O}_{2(q)}$ and $\mathbf{O}_{3(q)}$. The iteration continues till the stall condition is met.

\section{EXPERIMENTAL RESULTS}

In order to validate the proposed method, we test with data acquired from a HMC409LP4E PA fabricated by Analog Devices. Its nominal gain at $3.5 \mathrm{GHz}$ is $31 \mathrm{~dB}$ and the saturated output power is $32.5 \mathrm{dBm}$. We generate a stimulus at the carrier frequency of $3.5 \mathrm{GHz}$ in the PC Workstation and feed it to the PA through an Arbitrary Waveform Generator (AWG) with $10 \mathrm{GHz}$ sampling frequency. The input and output baseband signals of 15000 samples are time-aligned after downsampling to $120 \mathrm{MHz}$ for behavioral modeling. A $20 \mathrm{MHz}$ long term evolution (LTE) signal and a $40 \mathrm{MHz}$ wireless local area network (WLAN) signal are used as stimulus in the tests. Their peak-to-average power ratio (PAPR) are $8 \mathrm{~dB}$ and $12 \mathrm{~dB}$ respectively.

To determine the necessary number of training epochs with the aim of significantly reducing the search algorithm running time, we first study the convergence of the NN model training error as a function of the epoch number as depicted in Fig. 2. The training procedure is standard and uses the data randomly divided into a training set $(70 \%)$, a validation set $(15 \%)$ and a test set $(15 \%)$. The activation function is tansig. In order to study the separation of convergences of different NN models, we make the tests of a 1-hidden-layer (1HL) NN and a $2 \mathrm{HL}$ NN which have surely different performances [8]. For sake of the simplicity, we choose a $1 \mathrm{HL} \mathrm{NN}$ with $L=5, N_{1}=30$ to test. Since the NN is trained with $70 \%$ data randomly selected from the signal and the initial values of $w_{j k}$ and $b_{k}$ are random, its modeling accuracy is a random variable. We independently train and test the NN model for 50 times. With 50 realizations, we calculate the min, max, and average of their test errors at each epoch.

We can see that the performance convergence of these two NN models slows down after 10 epochs and a $2 \mathrm{HL}$ NN almost 
(a)

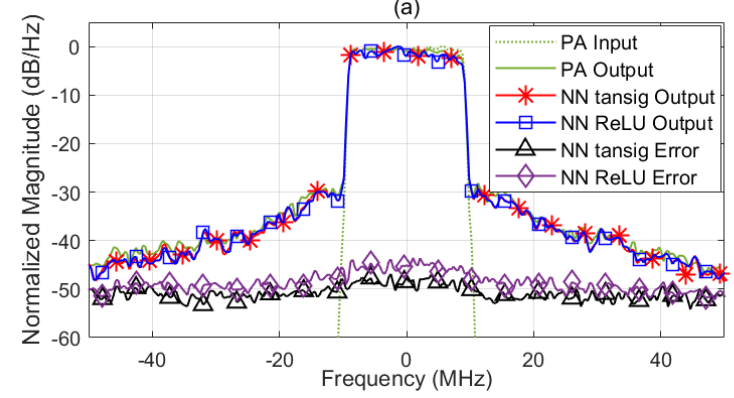

(c)

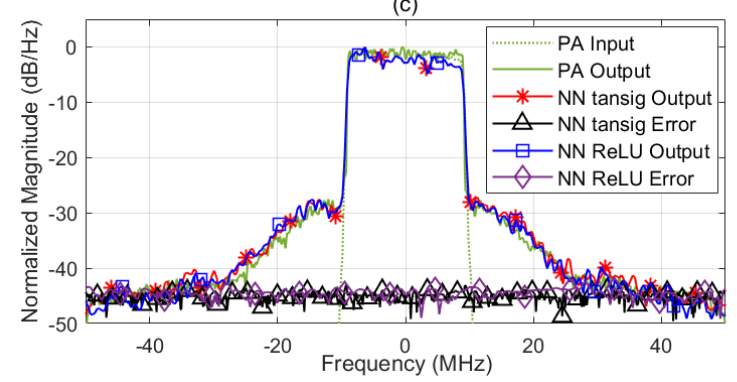

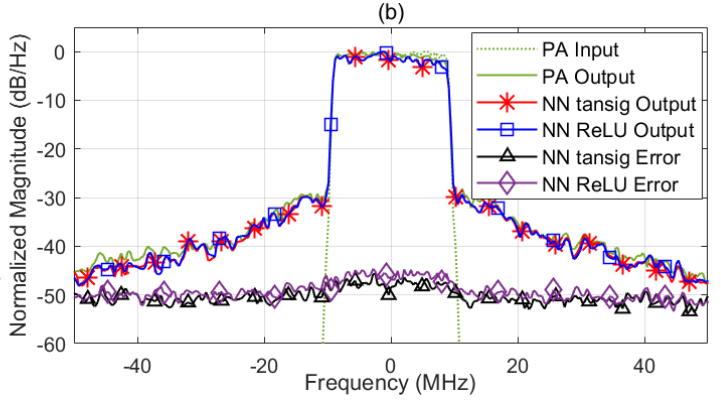

(d)

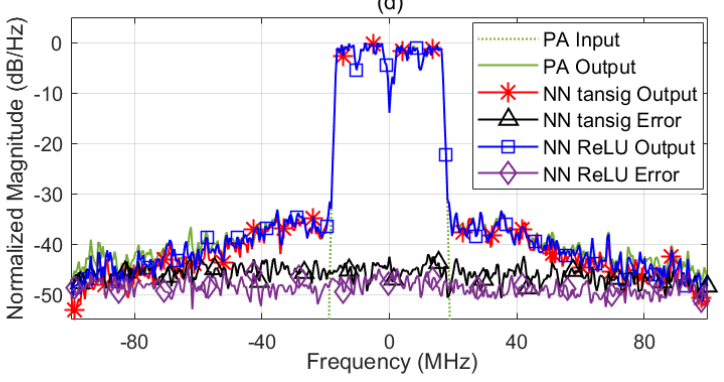

Fig. 3. Experimental results: (a) Output spectra of the PA and the determined NN model $M_{20}$ for 20 MHz LTE signal, (b) Output spectra of the PA and the determined NN model $M_{100}$ for $20 \mathrm{MHz}$ LTE signal, (c) Output spectra of the PA and the determined NN model $M_{20}$ for $20 \mathrm{MHz}$ LTE signal at saturation region, (d) Output spectra of the PA and the determined NN model for $40 \mathrm{MHz}$ WLAN signal

always outperforms a 1HL NN after 20 epochs. In other words, using a $2 \mathrm{HL} \mathrm{NN}$ model allows to significantly reduce the number of epochs needed to get the better performance/training time tradeoff. Since our objective is to find the optimal NN hyperparameters instead of giving the precise minimum error, we propose to reduce the epoch number to 20 in the GA search considering the variance of the NN performance.

In the search algorithm, we set the population size to 10 for each generation and the maximum number of generations to $Q=6$. When computing fitness, we evaluate the performance by training the corresponding NN model with 20 and 100 epochs separately.

Based on the data measured with the PA, we applied the proposed GA with 20 and 100 training epochs. The final solutions of the NN model determined by the GA are denoted by $M_{20}$ and $M_{100}$ respectively. We obtain their final performances by training the $M_{20}$ and $M_{100}$ with 1000 epochs. The output spectra of the optimal NN models trained with 1000 epochs are depicted in Fig.3(a) and Fig.3(b) respectively, as well as the corresponding modeling errors. The results in Fig.3(c) validate the NN model performance in saturation region by increasing the input power $2 \mathrm{~dB}$. We also make a test with $40 \mathrm{MHz}$ bandwidth WLAN signal as illustrated Fig.3(d), which confirms the effectiveness of our proposed method.

Table I gives the quantitative performances of NN models determined by our proposed method with LTE and WLAN signals. We take a generalized memory polynomial model as a reference whose optimal structure is determined using the method in [11]. With the LTE and WLAN signals, the NMSE values obtained are $-34.7 \mathrm{~dB}$ and $-37.0 \mathrm{~dB}$ respectively which are similar to the performance of the $\mathrm{NN}$ model determined by GA. The algorithm running time $T$ is measured under Matlab with an Intel(R) Core(TM) i7-3770 CPU @3.40GHz and 16GB RAM.

The values in "fitness" are the NMSE values between the
TABLE I

BEHAVIORAL MODELING PERFORMANCE OF NN GIVEN BY ALGORITHM 1

\begin{tabular}{|l|c|c|c|c|c|c|c|}
\hline Model & $\begin{array}{c}\text { Ac } \\
\text { Fcn }\end{array}$ & $L$ & $N_{1}$ & $N_{2}$ & $\begin{array}{c}T \\
(\mathrm{~min})\end{array}$ & $\begin{array}{c}\text { Fitness } \\
(\mathrm{dB})\end{array}$ & $\begin{array}{c}\text { NMSE } \\
(\mathrm{dB})\end{array}$ \\
\hline \multirow{2}{*}{$M_{20}$} & tansig & 7 & 46 & 49 & 151 & -54.1 & -33.7 \\
\cline { 2 - 8 } & ReLU & 7 & 48 & 32 & 124 & -54.5 & -33.3 \\
\hline \multirow{2}{*}{$M_{100}$} & tansig & 9 & 42 & 44 & 876 & -55.2 & -32.4 \\
\cline { 2 - 8 } & ReLU & 9 & 43 & 42 & 503 & -55.6 & -32.1 \\
\hline \multirow{2}{*}{$M_{\text {wlan } 20}$} & tansig & 9 & 26 & 37 & 121 & -48.4 & -34.2 \\
\cline { 2 - 8 } & ReLU & 10 & 48 & 33 & 110 & -49.5 & -36.1 \\
\hline
\end{tabular}

Ac Fen: Activation Function; T: Running time of algorithm

model output and measured PA output during the training process. The values in "NMSE" are the NMSE values obtained by testing the GA-determined model after 1000-epoch training. For the solution model determined by the GA, we calculate its average NMSE value with 50 tests. The proposed algorithm with 20 epochs seems to be a good tradeoff between modeling accuracy and running time. Increasing the number of epochs to 100 does not bring a sufficient improvement in performance while the running time is more than 4 times longer.

The activation function has impact on the algorithm running time. The search with ReLU needs less time since computational complexity of ReLU is lower than tansig. The performance of ReLU is better for WLAN signal.

\section{CONCLUSION}

In this paper, we propose a GA to optimize the hyperparameter of a NN-based PA behavioral model. To the authors' knowledge, this is the first time this optimization problem is addressed without exhaustive search. The algorithm is validated with measurement data acquired from a PA with different signals. We study the convergence of the NN model training error as a function of the epoch number and significantly reduce the optimization running time while keeping similar performances. Different activation functions of the NN model are tested. ReLU leads to less running time. 


\section{REFERENCES}

[1] F. M. Ghannouchi, "Power amplifier and transmitter architectures for software defined radio systems," IEEE Circuits Syst. Mag., vol. 10, no. 4, pp. 56-63, Fourthquarter 2010.

[2] C. Fager, T. Eriksson, F. Barradas, K. Hausmair, T. Cunha, and J. C. Pedro, "Linearity and efficiency in $5 \mathrm{~g}$ transmitters: New techniques for analyzing efficiency, linearity, and linearization in a $5 \mathrm{~g}$ active antenna transmitter context," IEEE Microw. Mag., vol. 20, no. 5, pp. 35-49, May 2019.

[3] F. Ghannouchi and O. Hammi, "Behavioral modeling and predistortion," IEEE Microw. Mag., vol. 10, no. 7, pp. 52 -64, dec. 2009.

[4] J. Cai, C. Yu, L. Sun, S. Chen, and J. B. King, "Dynamic behavioral modeling of rf power amplifier based on time-delay support vector regression," IEEE Trans. Microw. Theory Techn., vol. 67, no. 2, pp. 533-543, Feb 2019.

[5] T. Liu, S. Boumaiza, and F. Ghannouchi, "Dynamic behavioral modeling of $3 \mathrm{~g}$ power amplifiers using real-valued time-delay neural networks," IEEE Trans. Microw. Theory Techn., vol. 52, no. 3, pp. 1025-1033, March 2004

[6] F. Mkadem and S. Boumaiza, "Physically inspired neural network model for rf power amplifier behavioral modeling and digital predistortion," IEEE Trans. Microw. Theory Techn., vol. 59, no. 4, pp. 913-923, April 2011.

[7] D. Wang, M. Aziz, M. Helaoui, and F. M. Ghannouchi, "Augmented real-valued time-delay neural network for compensation of distortions and impairments in wireless transmitters," IEEE Trans. Neural Netw. Learn. Syst., vol. 30, no. 1, pp. 242-254, Jan 2019.

[8] P. Jaraut, M. Rawat, and F. M. Ghannouchi, "Composite neural network digital predistortion model for joint mitigation of crosstalk, $i / q$ imbalance, nonlinearity in mimo transmitters," IEEE Trans. Microw. Theory Techn., vol. 66, no. 11, pp. 5011-5020, Nov 2018.

[9] R. Hongyo, Y. Egashira, T. M. Hone, and K. Yamaguchi, "Deep neural network-based digital predistorter for doherty power amplifiers," IEEE Microw. Wireless Compon. Lett., vol. 29, no. 2, pp. 146-148, Feb 2019.

[10] B. Nakisa, M. N. Rastgoo, A. Rakotonirainy, F. Maire, and V. Chandran, "Long short term memory hyperparameter optimization for a neural network based emotion recognition framework," IEEE Access, vol. 6, pp. 49325-49338, 2018.

[11] S. Wang, M. Abi Hussein, O. Venard, and G. Baudoin, "A novel algorithm for determining the structure of digital predistortion models," IEEE Trans. Veh. Technol., vol. 67, no. 8, pp. 7326-7340, Aug 2018.

[12] S. Wang, M. Abi Hussein, O. Venard, and G. Baudoin, "Optimal sizing of two-stage cascaded sparse memory polynomial model for high power amplifiers linearization," IEEE Trans. Microw. Theory Techn., vol. 66, no. 9, pp. 3958-3965, Sep. 2018.

[13] A. Abdelhafiz, L. Behjat, and F. M. Ghannouchi, "Generalized memory polynomial model dimension selection using particle swarm optimization," IEEE Microw. Wireless Compon. Lett., vol. 28, no. 2, pp. 96-98, Feb 2018.

[14] M. T. Hagan and M. B. Menhaj, "Training feedforward networks with the marquardt algorithm," IEEE Trans. Neural Netw., vol. 5, no. 6, pp. 989-993, Nov 1994. 